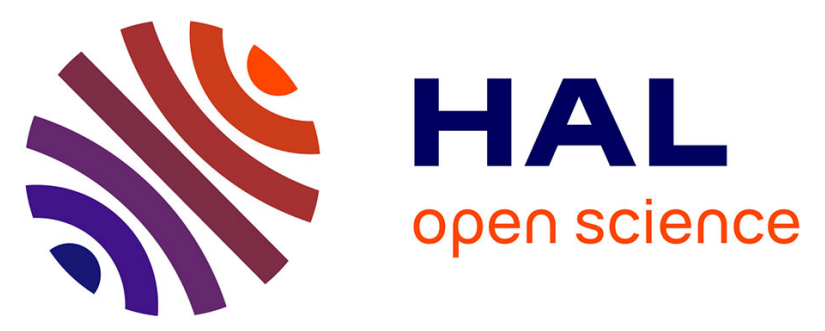

\title{
Robotic Test Bench for CubeSat Ground Testing: Concept and Satellite Dynamic Parameter Identification
}

Irina Gavrilovich, Sébastien Krut, Marc Gouttefarde, François Pierrot, Laurent Dusseau

\section{- To cite this version:}

Irina Gavrilovich, Sébastien Krut, Marc Gouttefarde, François Pierrot, Laurent Dusseau. Robotic Test Bench for CubeSat Ground Testing: Concept and Satellite Dynamic Parameter Identification. IROS: Intelligent RObots and Systems, Sep 2015, Hamburg, Germany. pp.5447-5453, 10.1109/IROS.2015.7354148 . lirmm-01310817

\section{HAL Id: lirmm-01310817 https://hal-lirmm.ccsd.cnrs.fr/lirmm-01310817}

Submitted on 3 May 2016

HAL is a multi-disciplinary open access archive for the deposit and dissemination of scientific research documents, whether they are published or not. The documents may come from teaching and research institutions in France or abroad, or from public or private research centers.
L'archive ouverte pluridisciplinaire HAL, est destinée au dépôt et à la diffusion de documents scientifiques de niveau recherche, publiés ou non, émanant des établissements d'enseignement et de recherche français ou étrangers, des laboratoires publics ou privés. 


\title{
Robotic Test Bench for CubeSat Ground Testing: Concept and Satellite Dynamic Parameter Identification*
}

\author{
Irina Gavrilovich ${ }^{1}$, Sébastien Krut ${ }^{1}$, Marc Gouttefarde ${ }^{1}$, François Pierrot ${ }^{1}$, and Laurent Dusseau ${ }^{2}$
}

\begin{abstract}
This paper introduces a novel concept of an air bearing test bench for CubeSat ground testing together with the corresponding dynamic parameter identification method. Contrary to existing air bearing test benches, the proposed concept allows three degree-of-freedom unlimited rotations and minimizes the influence of the test bench on the tested CubeSat. These advantages are made possible by the use of a robotic wrist which rotates air bearings in order to make them follow the CubeSat motion. Another keystone of the test bench is an accurate balancing of the tested CubeSat. Indeed, disturbing factors acting on the satellite shall be minimized, the most significant one being the gravity torque. An efficient balancing requires the CubeSat center of mass position to be accurately known. Usual techniques of dynamic parameter identification cannot be directly applied because of the frictionless suspension of the CubeSat in the test bench and, accordingly, due to the lack of external actuation. In this paper, a new identification method is proposed. This method does not require any external actuation and is based on the sampling of free oscillating motions of the CubeSat mounted on the test bench.
\end{abstract}

\section{INTRODUCTION}

One of the widespread and demanded branches of space technologies is CubeSat-class satellites. CubeSats are miniaturized satellites with standardized sizes of one-unit $(1 \mathrm{U}, 10 \times 10 \times 10 \mathrm{~mm})$, or a multiple of one unit (e.g. 3U). The development of such satellites is common in schools and universities due to the relatively short development time, the wide range of components available off-the-shelf, and compatibility with standardized launch ejectors. In addition to educational purposes, nowadays, CubeSats are employed in full scale scientific and technological missions, and every year the number of professional CubeSat programs increases [1]. This tendency leads to the necessity of complete CubeSat ground testing. One of the main issues of the pre-launch examination is the inspection of the Attitude Determination and Control System (ADCS) once it is assembled and integrated in the satellite. ADCS testing, unlike tests of other subsystems, requires the satellite to move. Each constraint impairing the satellite free rotation yields cases where the ADCS cannot be checked and might thus cause problems during its on-orbit operation. Results obtained for the available range of angles are often extrapolated to other

\footnotetext{
* Research supported by Van Allen Foundation and FEDER.

1 I. Garilovich, S. Krut, M. Gouttefarde, and F. Pierrot are with the Montpellier Laboratory of Informatics, Robotics and Microelectronics (LIRMM), University of Montpellier and CNRS, 34090 Montpellier, France (e-mails: irina.gavrilovich/sebastien.krut/ marc.gouttefarde/francois.pierrot@lirmm.fr).

${ }^{2}$ L. Dusseau is with the Centre Spatial Universitaire (CSU), University of Montpellier, $34090 \quad$ Montpellier, France (e-mails: laurent.dusseau@ies.univ-montp2.fr)
}

attitudes in order to confirm ADCS operability, which decreases the probability of faultless operation.

The development of an ADCS test bench is a challenging task, but some examples can be found in the literature. Most of the developed test benches are designed for satellites with a mass greater than $50 \mathrm{~kg}$ [2]-[9]. They cannot be used for CubeSat ADCS tests, because inertia parameters of the test bench are critical for a reliable checkout and shall be comparable with those of the tested satellite. Some test benches have been designed specifically for CubeSats [10][12], but their maximum rotation angle is approximately $90^{\circ}$ or less.

In this paper, a novel approach to the design of $1 . .3 \mathrm{U}$ CubeSat test benches is proposed. The corresponding design eliminates the limitations on the satellite attitude (orientation) during the testing since it allows full three degree-of-freedom (DoF) satellite rotations. The test bench consists of two frames, which have spherical mating surfaces conventionally called Spheres, and of a 4 DoF redundant robotic wrist. Moreover, four air bearings are employed to allow frictionless motion of the satellite mounted on the test bench.

Reliable and trustworthy results of ADCS testing can be obtained only if the effects of all disturbing torques caused by gravity, aerodynamic drag, and friction, are minimized. The total disturbing torque shall be one order of magnitude smaller than the total expected external torque on the orbit [8]. While other sources of disturbances can be reduced up to negligible values by optimizing environmental conditions at the test facility, the torque due to gravity always influences the satellite when there is an offset between the satellite center of mass $(\mathrm{CM})$ and the test bench center of rotation (CR). A balancing procedure, aiming at eliminating this offset, requires an accurate identification of the satellite $\mathrm{CM}$ position together with its inertial parameters. In the sequel, the inertial parameters and the CM position are referred to as the dynamic parameters.

The identification of dynamic parameters is a well-known technique in robotics and related fields. Several approaches have been proposed in the literature to solve the dynamic parameter identification problem [2], [13]-[18]. Some common features of these approaches can be found in [15], [18], namely:

- The use of inverse dynamic or energy model to form the identification equations;

- The use of an optimal exciting trajectory for efficient model sampling;

- The use of an over-determinate linear system of equations resulting from the model sampling;

- Solving the linear system by the Least Squares method to estimate the parameters.

In all the aforementioned approaches, the identification is done for systems subjected to external actuation (usually, 
joint forces/torques). For example in [2], where the identification and balancing of an $800 \mathrm{~kg}$ satellite is done, the test bench uses reaction wheels for actuation. The identification equations can be written in the following general form

$$
\mathbf{y}(\boldsymbol{\Gamma})=\mathbf{W} \mathbf{x}
$$

where $\boldsymbol{\Gamma}$ is an external torque, $\mathbf{W}$ is an observation matrix, and $\mathbf{x}$ is the vector of the dynamic parameters to be identified. In the case of a passive system (with no actuation), the right-hand side of (1) is always equal to zero. The latter case is the one dealt with in this paper where the identification problem concerns the payload of the test bench which is passive, i.e., it is not subjected to external influences apart from the torque due to gravity. A method of dynamic parameter identification suitable to this passive case and based on the sampling of free oscillating rotations is presented in this paper.

In summary, the first contribution of this paper is a novel design of an air bearing test bench for CubeSat ADCS testing, which includes a 4-DoF redundant robotic wrist. The second contribution is a dynamic parameter identification technique for a passive system. This technique is based on the observation of its oscillating rotational motions.

The paper is organized as follows: Section 2 introduces the details of the test bench design, Sections 3 and 4 are devoted to the identification method. Section 5 presents simulation results while section 6 concludes the paper.

\section{The TeSt BENCH}

The most popular and advanced technique to obtain frictionless 3-DoF rotational motions of a satellite on a test bench is the use of air bearings. Different types of air bearing test benches are discussed in [19], but all of these designs have limitations on the available pitch and roll rotations of the mounted payload, the maximum rotation amplitudes being around $\pm 45^{\circ}$. These limitations could be eliminated if the payload is placed at the center of a hollow sphere gliding on an air bearing puck. Such an approach was tried for satellite disassembled hardware, the system provided rotations of $\pm 180^{\circ}$ but the reported tests included single-axis rotations only [20]. In [9], an air-levitated sphere with a $1 \mathrm{U}$ CubeSat placed inside is described, but it was not realized because of technological issues. A hollow sphere able to hold 3U CubeSats would be bulky and heavy, which are undesirable characteristics for a CubeSat test bench. Another disadvantage is technological complexity: The hollow sphere shall be built in several sections to be able to place a payload inside the sphere, while the surface shall be accurately machined to provide the roughness required by the air bearings. The design proposed in this paper has the advantage of the air-levitated sphere (satellite unconstrained rotations) without its aforementioned weaknesses.

\section{A. Concept}

The key advantage of the hollow sphere is the continuous surface that allows friction-free contact with the bearing puck at every possible angular position. The idea proposed here is that the same property can be achieved by means of

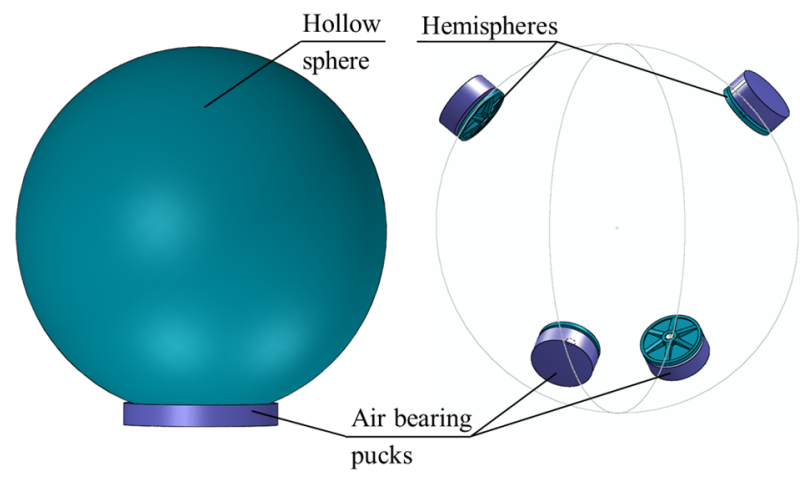

Figure 1. An air-levitated sphere and a spherical structure of the same diameter formed by several air bearings.

several small air bearings rotating around a common CR placed at the spherical surface center (Fig. 1). The inner part of the structure, which consists of small hemispheres (spherical segments) as shown in the right part of Fig. 1, becomes much smaller and lighter than the air-levitated spheres suggested before. It is relevant to minimize the size and mass of this part of the test bench because of its unwanted moment of inertia. The frame on which the bearing pucks are attached, called the "External Sphere", needs to follow the motion of the fictitious "Inner Sphere" wherein the satellite is mounted. The External Sphere is independent from the CubeSat mounted on the test bench and, therefore, has no influence on results of the tests.

\section{B. Design overview}

The proposed CubeSat test bench mainly consists of three parts: (i) the Inner Sphere composed of the sliding hemispheres attached to the CubeSat; (ii) the External Sphere built of the air bearing pucks; and (iii) a 4 DoF redundant robotic wrist, able to rotate the External Sphere around the CR (Figure 2). The External Sphere shall be rotated around the CR in order to follow the motion of the Inner Sphere so that each hemisphere remains aligned with its air bearing puck. The CR is thus common to both the

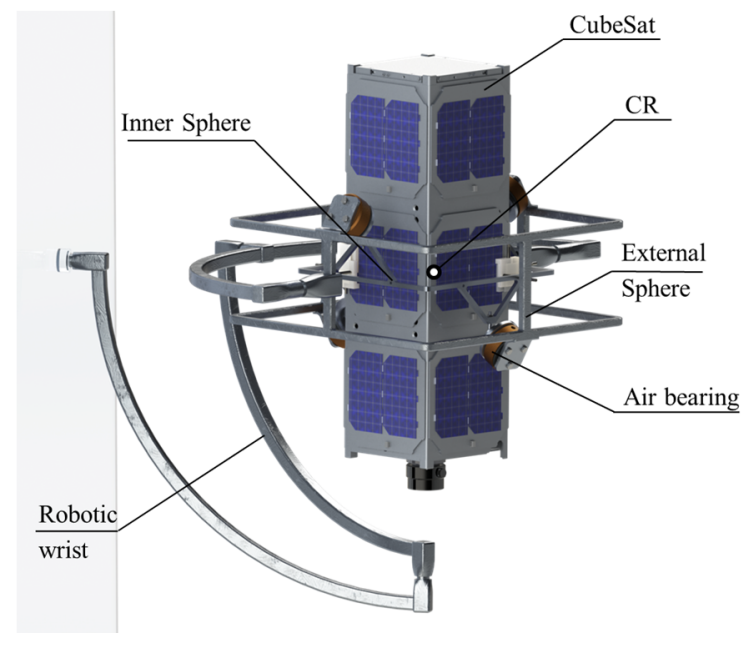

Figure 2. The test bench. 
Inner and External Spheres and is coincident with the common intersection point of the four robotic wrist revolute joint axes. While the whole system needs only 3 rotational DoF, the additional DoF of the wrist aims at eliminating kinematic singularities which would limit the possible rotations.

The CubeSat is fixed to the Inner Sphere by means of an adjusting mechanism, which allows limited modifications of the satellite position with respect to the CR. This feature is needed for sampling the payload motion with different initial offsets between the $\mathrm{CR}$ and the $\mathrm{CM}$ and for the final balancing of the CubeSat. The maximum displacements of the satellite inside the Inner Sphere are $\pm 20 \mathrm{~mm}$ along the $X$ and $\mathrm{Y}$ directions and $\pm 35 \mathrm{~mm}$ in the $\mathrm{Z}$ direction. These values have been chosen according to CubeSat Design Specification [21] that defines allowable CM locations for 1 ...3U CubeSats.

The motion of the satellite is a key point of ADCS tests and shall not be predicted but only observed. Moreover, the positioning of the External Sphere has to be corrected rapidly based on the current position of the satellite. For these purposes, a tracking system is required. Two possible means to observe the Inner Sphere motion are: 3D motion capture cameras or distance sensors. The sensors measure a relative position of the hemispheres and pucks. In order to obtain an absolute estimation of the Inner Sphere attitude, the encoders of the robotic wrist joints are then required.

\section{The Dynamics of the Test Bench PAyloAD}

The payload of the test bench is the CubeSat together with the Inner Sphere and the adjusting mechanism, which are rigidly connected and move as one body. Before dynamic parameter identification, the dynamics of the payload shall be described. In the sequel, "body" refers to the payload.

\section{A. Frames}

The inertial fixed frame $\mathcal{R}_{I}$ is defined by the basis $\mathcal{B}_{I}$, denoted $\left(\mathbf{x}_{\mathbf{I}}, \mathbf{y}_{\mathbf{I}}, \mathbf{z}_{\mathbf{I}}\right)$, and its origin centered at the point $C R$. Vector $\mathbf{z}_{\mathbf{I}}$ is aligned with the local vertical (Fig. 3). Let the basis $\mathcal{B}_{b f}$, denoted $(\mathbf{x}, \mathbf{y}, \mathbf{z})$, be attached to the body, and let the body-fixed reference frame $\mathcal{R}_{b f}$ consist of $\mathcal{B}_{b f}$ centered at a given point $O$ of the body. The choice of $O$ will be discussed in Section 4.

The frame $\mathcal{R}_{B F}$ shown in Fig. 3 has the same orientation as $\mathcal{B}_{b f}$ but its origin is $C R$. As shown in the sequel, the

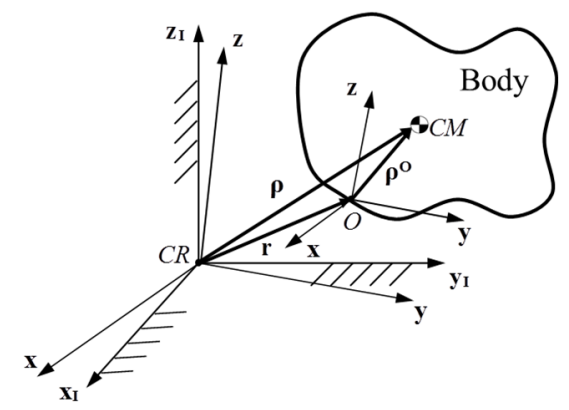

Figure 3 . The frames involved in the identification process. frame $\mathcal{R}_{B F}$ is introduced to simplify the writing of the equations of motion, suitable for identification. The position of the point $C M$ in $\mathcal{R}_{B F}$ is defined by the column vector $\boldsymbol{\rho}$. As shown in Fig. 3, it can be written as the sum of the vectors $\boldsymbol{\rho}^{\mathbf{o}}$ and $\mathbf{r}$ expressed in $\mathcal{B}_{b f}$

$$
\boldsymbol{\rho}=\mathbf{r}+\boldsymbol{\rho}^{\mathbf{o}}
$$

It should be noted that the vector $\mathbf{r}$ is related to the position of the body in $\mathcal{R}_{B F}$ and the vector $\boldsymbol{\rho}^{\mathbf{o}}$ is constant for the given body (Fig. 3).

\section{B. Kinematics}

The orientation of $\mathcal{R}_{B F}$ with respect to $\mathcal{R}_{I}$ is given by the rotation matrix ${ }^{\mathbf{I}} \mathbf{A}_{\mathbf{B F}}$. In $\mathcal{B}_{I}$, the angular velocity vector of the body ${ }^{\mathrm{I}} \boldsymbol{\omega}$ can be found as follows

$$
\left[{ }^{\mathbf{I}} \boldsymbol{\omega}\right]_{\times}={ }^{\mathbf{I}} \dot{\mathbf{A}}_{\mathbf{B F}}{ }^{\mathbf{I}} \mathbf{A}_{\mathbf{B F}}^{T}
$$

where $\left[{ }^{\mathrm{I}} \boldsymbol{\omega}\right]_{\times}$is the skew-symmetric matrix associated to the vector $^{\mathrm{I}} \boldsymbol{\omega}$. The following operation can be used to express the angular velocity vector $\omega$ in $\mathcal{B}_{b f}$

$$
\omega={ }^{B F} \mathbf{A}_{\mathbf{I}}{ }^{\mathrm{I}} \boldsymbol{\omega}
$$

In the sequel, all vectors without a left superscript are expressed in $\mathcal{B}_{b f}$.

\section{Dynamics}

The Euler's equations of motion traditionally describes the rotational dynamics of a body with respect to a coordinate frame whose origin is the body's CM. The test bench payload is subjected to the action of a gravity torque (when the points $C R$ and $C M$ are not perfectly coincident) and to the reaction forces at the air bearings. The reaction forces are pointing towards $C R$. They can be represented by a resulting force passing through $C R$. The magnitude and direction of this force are unknown.

However, the equations of motion does not include this unknown resulting force if the Euler's equations of motion are expressed in $\mathcal{R}_{B F}$

$$
\mathrm{I}^{\mathrm{CR}} \dot{\boldsymbol{\omega}}+\omega \times \mathrm{I}^{\mathrm{CR}} \omega=\mathrm{T}^{\mathrm{CR}}
$$

where $\mathbf{I}^{\text {CR }}$ is the inertia matrix of the body at $C R, \mathbf{T}^{\text {CR }}$ is the torque induced at $C R$ by the weight $m \mathbf{g}$ of the body

$$
\mathbf{T}^{\mathbf{C R}}=\boldsymbol{\rho} \times m \mathbf{g}=-m[\mathbf{g}]_{\times} \boldsymbol{\rho}=-m\left[{ }^{\mathbf{B F}} \mathbf{A}_{\mathbf{I}}{ }^{\mathbf{I}} \mathbf{g}\right]_{\times} \boldsymbol{\rho}
$$

Substituting (6) into (5) results in

$$
\mathbf{I}^{\mathrm{CR}} \dot{\boldsymbol{\omega}}+\boldsymbol{\omega} \times \mathbf{I}^{\mathrm{CR}} \boldsymbol{\omega}+m\left[{ }^{\mathrm{BF}} \mathbf{A}_{\mathbf{I}}{ }^{\mathrm{I}} \mathbf{g}\right]_{\times} \boldsymbol{\rho}=\mathbf{0}
$$

Equation (7) describes the dynamics of the test bench payload in $\mathcal{B}_{b f}$. As detailed in Section 4, this equation can 
be used to obtain the identification equations in the following form

$$
\Phi \mathbf{x}=\mathbf{b}
$$

where vector $\mathbf{x}$ contains the body dynamic parameters to be identified.

\section{IDENTIFICATION OF THE DYNAMIC PARAMETERS}

The goal of the identification process is to find the dynamic parameters of the body. The dynamic model (7) in the current formulation is a function of these parameters taken with respect to $C R$ ( $\mathbf{I}^{\mathbf{C R}}$ and $\boldsymbol{\rho}$ ) so that the vector $\mathbf{b}$ in (8) is always equal to zero. Moreover, $\mathbf{I}^{\mathbf{C R}}$ and $\boldsymbol{\rho}$ are related to the position of the body in $\mathcal{R}_{B F}$, hence the result of the identification depends on the initial position of the body with respect to $C R$. Such an objectionable situation can be avoided if the dynamic parameters are taken at a point attached to the body. The points $C M$ and $O$ (Fig. 3) are two candidate points. Choosing $C M$ yields nonlinearities in the dynamic parameters. On the contrary, choosing $O$ leads to a linear system. $\mathbf{I}^{\mathbf{C R}}$ and $\boldsymbol{\rho}$ shall thus be expressed with respect to $O$ and substituted into (7).

\section{A. The Inertia Matrix}

The inertia matrix $\mathbf{I}^{\mathbf{C M}}$ taken at $C M$ and expressed in $\mathcal{B}_{b f}$ is:

$$
\mathbf{I}^{\mathbf{C M}}=\left[\begin{array}{lll}
I_{x x}^{C M} & I_{x y}^{C M} & I_{x z}^{C M} \\
I_{x y}^{C M} & I_{y y}^{C M} & I_{y z}^{C M} \\
I_{x z}^{C M} & I_{y z}^{C M} & I_{z z}^{C M}
\end{array}\right]
$$

Then, I ${ }^{\mathbf{C R}}$ can be found by applying the HuygensSteiner theorem:

$$
\mathbf{I}^{\mathbf{C R}}=\mathbf{I}^{\mathbf{C M}}+m\left(\boldsymbol{\rho}^{T} \boldsymbol{\rho} \mathbf{1}-\boldsymbol{\rho} \boldsymbol{\rho}^{T}\right)
$$

where $\mathbf{1}$ is the $3 \times 3$ identity matrix. Substituting (2) into (10) gives

$$
\begin{aligned}
\mathbf{I}^{\mathbf{C R}} & =\mathbf{I}^{\mathbf{C M}}+m\left(\left(\boldsymbol{\rho}^{\mathbf{o}}+\mathbf{r}\right)^{T}\left(\boldsymbol{\rho}^{\mathbf{o}}+\mathbf{r}\right) \mathbf{1}-\left(\boldsymbol{\rho}^{\mathbf{o}}+\mathbf{r}\right)\left(\boldsymbol{\rho}^{\mathbf{o}}+\mathbf{r}\right)^{T}\right) \\
& =\mathbf{I}^{\mathbf{O}}+\mathbf{C}(\mathbf{r})+m\left(2 \boldsymbol{\rho}^{\mathbf{o} T} \mathbf{r} \mathbf{1}-\left(\boldsymbol{\rho}^{\mathbf{o}} \mathbf{r}^{T}+\mathbf{r} \boldsymbol{\rho}^{\mathbf{o} T}\right)\right)
\end{aligned}
$$

where

$$
\mathbf{I}^{\mathbf{O}}=\mathbf{I}^{\mathbf{C M}}+m\left(\boldsymbol{\rho}^{\mathbf{O} T} \boldsymbol{\rho}^{\mathbf{O}} \mathbf{1}-\boldsymbol{\rho}^{\mathbf{O}} \boldsymbol{\rho}^{\mathbf{O} T}\right)
$$

is the inertia matrix of the body taken at $O$ and expressed in $\mathcal{B}_{b f}$ and

$$
\mathbf{C}(\mathbf{r})=m\left(\mathbf{r}^{T} \mathbf{r} \mathbf{1}-\mathbf{r} \mathbf{r}^{T}\right)
$$

is a value depending only on the position of the body in $\mathcal{R}_{B F}$ and on the body's mass $m$. The decomposition of the inertia matrix presented in (11) is convenient for further simplification and transformation of (7).

\section{B. The Identification Equations}

Taking into account the relations obtained in the previous section, (7) can be rewritten as a linear equation in the dynamic parameters. The product of the inertia matrix and the angular velocity becomes

$$
\mathbf{I}^{\mathrm{CR}} \boldsymbol{\omega}=\mathbf{I}^{\mathbf{O}} \boldsymbol{\omega}+\mathbf{C}(\mathbf{r}) \boldsymbol{\omega}+m \mathbf{B}(\boldsymbol{\omega}, \mathbf{r}) \boldsymbol{\rho}^{\mathbf{O}}
$$

where matrix $\mathbf{B}(\boldsymbol{\omega}, \mathbf{r})$ is defined as

$$
\mathbf{B}(\boldsymbol{\omega}, \mathbf{r})=\left[\begin{array}{ccc}
-r_{2} \omega_{2}-r_{3} \omega_{3} & 2 r_{2} \omega_{1}-r_{1} \omega_{2} & 2 r_{3} \omega_{1}-r_{1} \omega_{3} \\
2 r_{1} \omega_{2}-r_{2} \omega_{1} & -r_{1} \omega_{1}-r_{3} \omega_{3} & 2 r_{3} \omega_{2}-r_{2} \omega_{3} \\
2 r_{1} \omega_{3}-r_{3} \omega_{1} & 2 r_{2} \omega_{3}-r_{3} \omega_{2} & -r_{1} \omega_{1}-r_{2} \omega_{2}
\end{array}\right] \text { (15) }
$$

Equation (14) can be modified to highlight the fact that it is linear in the unknown values $\mathbf{I}^{\mathbf{O}}$ and $\boldsymbol{\rho}^{\mathbf{o}}$. The first term on the right-hand side can be written

$$
\mathbf{I}^{\mathrm{o}} \omega=\Omega(\omega) \mathbf{j}^{\mathrm{o}}
$$

where $\mathbf{j}^{\mathbf{0}}$ is a $6 \times 1$ vector composed of the elements of the inertia matrix $\mathbf{I}^{\mathbf{O}}$ and $\boldsymbol{\Omega}(\boldsymbol{\omega})$ is a $3 \times 6$ matrix composed of the elements of the vector $\boldsymbol{\omega}$

$$
\begin{gathered}
\mathbf{j}^{\mathbf{O}}=\left[\begin{array}{llllll}
I_{x x}^{O} & I_{y y}^{O} & I_{z z}^{O} & I_{x y}^{O} & I_{x z}^{O} & I_{y z}^{O}
\end{array}\right]^{T} \\
\boldsymbol{\Omega}(\boldsymbol{\omega})=\left[\begin{array}{cccccc}
\omega_{1} & 0 & 0 & \omega_{2} & \omega_{3} & 0 \\
0 & \omega_{2} & 0 & \omega_{1} & 0 & \omega_{3} \\
0 & 0 & \omega_{3} & \omega_{1} & \omega_{2} & 0
\end{array}\right]
\end{gathered}
$$

Accordingly, the expression of $\mathbf{I}^{\mathbf{C R}} \dot{\boldsymbol{\omega}}$ can be written as

$$
\mathbf{I}^{\mathrm{CR}} \dot{\boldsymbol{\omega}}=\mathbf{\Omega}(\dot{\boldsymbol{\omega}}) \mathbf{j}^{\mathrm{o}}+m \mathbf{B}(\dot{\boldsymbol{\omega}}, \mathbf{r}) \boldsymbol{\rho}^{\mathrm{o}}+\mathbf{C}(\mathbf{r}) \dot{\boldsymbol{\omega}}
$$

Collecting all dynamic parameters on the left-hand side, (7) is finally written in matrix form as

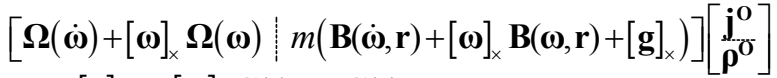

$$
\begin{aligned}
& =-m[\mathbf{g}]_{\times} \mathbf{r}-[\boldsymbol{\omega}]_{\times} \mathbf{C}(\mathbf{r}) \boldsymbol{\omega}-\mathbf{C}(\mathbf{r}) \dot{\boldsymbol{\omega}}
\end{aligned}
$$

\section{The Least Squares Solution}

In this work, the identification of the components of the inertia matrix and of the position of $C M$ is based on the knowledge of the angular position of the body-fixed frame with respect to $\mathcal{R}_{I}$. A Least Squares (LS) solution requires data obtained from $p$ experiments each having a duration of $N_{i}$ sec or $n_{i}$ time steps. $N_{i}$ and $p$ as well as the initial conditions of the experiments should be optimized in order to obtain the best results. During the experiments, the body is moving freely under the influence of the gravity torque created by the body weight when $C M$ is not coincident with $C R$. In each experiment, the initial conditions, i.e. the offset between $C R$ and $O$, described by the vector $\mathbf{r}_{i}$, the initial orientation of the body in $\mathcal{R}_{I}$ and its initial angular velocity could be different.

For the $j^{\text {th }}$ measurement of the $i^{\text {th }}$ experiment, (20) is

$$
\begin{aligned}
& {\left[\boldsymbol{\Omega}(\dot{\boldsymbol{\omega}})_{i j}+\left[\boldsymbol{\omega}_{i j}\right]_{x} \boldsymbol{\Omega}(\boldsymbol{\omega})_{i j}: m\left(\mathbf{B}\left(\dot{\boldsymbol{\omega}}_{i j}, \mathbf{r}_{i}\right)+\left[\boldsymbol{\omega}_{i j}\right]_{\times} \mathbf{B}\left(\boldsymbol{\omega}_{i j}, \mathbf{r}_{i}\right)+\left[\mathbf{g}_{i j}\right]_{\times}\right)\right]\left[\begin{array}{l}
\mathbf{j}^{\mathbf{O}} \\
\boldsymbol{\rho}^{\mathbf{O}}
\end{array}\right](21)} \\
& =-m\left[\mathbf{g}_{i j}\right]_{\times} \mathbf{r}_{i}-\left[\boldsymbol{\omega}_{i j}\right]_{\times} \mathbf{C}\left(\mathbf{r}_{i}\right) \boldsymbol{\omega}_{i j}-\mathbf{C}\left(\mathbf{r}_{i}\right) \dot{\boldsymbol{\omega}}_{i j}
\end{aligned}
$$


where $i=1 \ldots p, j=1 \ldots n_{i}$.

Finally, gathering all the measurements of all the experiments in a single equation gives

$$
\Phi_{\mathbf{X}}=\mathbf{b}
$$

where $\boldsymbol{\Phi}$ is a $3 n p \times 9$ observation matrix, $\mathbf{x}$ is the $9 \times 1$ vector of dynamic parameters and $\mathbf{b}$ is a $3 n p \times 1$ observation vector.

Since there are more equations than unknowns, this problem can be solved computing the LS solution

$$
\mathbf{x}=\boldsymbol{\Phi}^{+} \mathbf{b}
$$

where $\boldsymbol{\Phi}^{+}$is the pseudo-inverse of the observation matrix. This LS solution is an estimation of the dynamic parameters $\mathbf{x}$. The Huygens-Steiner theorem can finally be applied to find the inertia matrix of the body at its $C M$

$$
\mathbf{I}^{\mathbf{C G}}=\mathbf{I}^{\mathbf{O}}-m\left(\boldsymbol{\rho}^{\mathbf{o} T} \mathbf{\rho}^{\mathbf{O}} \mathbf{1}-\boldsymbol{\rho}^{\mathbf{O}} \boldsymbol{\rho}^{\mathbf{O} T}\right)
$$

\section{Iterative Approach for Multi-Body Payload}

The equations in the previous subsections are given for a single rigid body. Practically, in the test bench studied in this paper, the "body" consists of the CubeSat, the Inner Sphere and the adjusting mechanism. The identification shall be done only for the CubeSat's dynamic parameters, which are constant since the CubeSat does not change a shape during the experiments. The parameters of the other elements are assumed to be well known. While they are not a subject of interest here, they influence the motion and shall thus be taken into account. Accordingly, (2) becomes

$$
\boldsymbol{\rho}=\mathbf{r}+\frac{m}{m+\sum_{k=1}^{l} m_{k}} \boldsymbol{\rho}^{\mathbf{o}}+\frac{1}{m+\sum_{k=1}^{l} m_{k}} \sum_{k=1}^{l} m_{k} \boldsymbol{\rho}_{k}^{\mathbf{o}}
$$

where $m$ and $\boldsymbol{\rho}^{\mathbf{o}}$ refer now to the CubeSat while $m_{k}$ and $\boldsymbol{\rho}_{k}^{\mathbf{o}}$ refer to the elements attached to the satellite, $k=1 \ldots l$. Moreover, (13) becomes

$$
\mathbf{C}(\mathbf{r})=m\left(\mathbf{r}^{T} \mathbf{r} \mathbf{1}-\mathbf{r} \mathbf{r}^{T}\right)+\sum_{k=1}^{l} \mathbf{I}_{k}^{\mathbf{C R}}
$$

The resulting identification equations can be obtained by substituting (25) and (26) into (21).

Before a direct application of (21) to the identification of dynamic parameters, it should be mentioned that the vector $\mathbf{x}$ is not homogeneous. The components of $\mathbf{x}$ have different units (unit of $\boldsymbol{\rho}^{\mathbf{o}}$ is $\mathrm{m}$ and unit of $\mathbf{j}^{\mathbf{0}}$ is $\mathrm{kg} \cdot \mathrm{m}^{2}$ ). For CubeSats, the values of $\boldsymbol{\rho}^{\mathbf{o}}$ can be up to 2 orders of magnitude larger than the diagonal components of the inertia matrix, depending on the position of the $\mathrm{CM}$ and on the choice of the point $O$ on the body. An accurate identification can be reached when all the components of $\mathbf{x}$ are of the same order of magnitude. To this end, an iterative approach aiming to minimize the magnitude of $\boldsymbol{\rho}^{\mathbf{O}}$ can be considered. The following sequence of operations is suggested:

- Initialization: Choose the origin $O$ of $\mathcal{R}_{b f}$ at the geometric center of the CubeSat (as a first approximation of the CM position) and select the initial position of the CubeSat in $\mathcal{R}_{I}$ (i.e. vector $\mathbf{r}$ ) for each one of the $p$ experiments;

- Run $p$ experiments in order to form an overdeterminate linear system of equations (22);

- Derive $\mathbf{x}$ using the LS method;

- Update the position of $O$ in $\mathcal{R}_{I}$ according to the identified value $\boldsymbol{\rho}^{\mathbf{0}}$;

- Update $\mathbf{r}$ based on given scaling coefficients;

- Repeat the operations until the required accuracy is reached

This iterative approach results in decreasing the magnitude of $\rho^{\mathbf{o}}$ at every step. It can be seen in (12) that the contribution of $\mathbf{I}_{0}^{\mathbf{C G}}$ in $\mathbf{I}^{\mathbf{O}}$ is larger than the addition, which depends on $\boldsymbol{\rho}^{\mathbf{o}}$, because $\boldsymbol{\rho}^{\mathbf{o}}$ is small and squared. While $\boldsymbol{\rho}^{\mathbf{o}}$ decreases fast, $\mathbf{j}^{\mathbf{0}}$ changes slowly. Accordingly, after a few iterations, the values of $\boldsymbol{\rho}^{\mathbf{o}}$ and $\mathbf{j}^{\mathbf{0}}$ will be of the same order of magnitude and the identification error should be minimized. Besides, when $\boldsymbol{\rho}^{\mathbf{o}}$ is decreasing, the oscillation period increases and the experiment duration $N_{i}$ might not be large enough to capture the character of the motion. Consequently, a scaling of $N_{i}$ after each iteration is recommended to avoid this issue.

\section{MATLAB SIMULATION}

The identification algorithm proposed above shall be tested and, if required, improved before its application to the test bench. Simulations of the identification process in

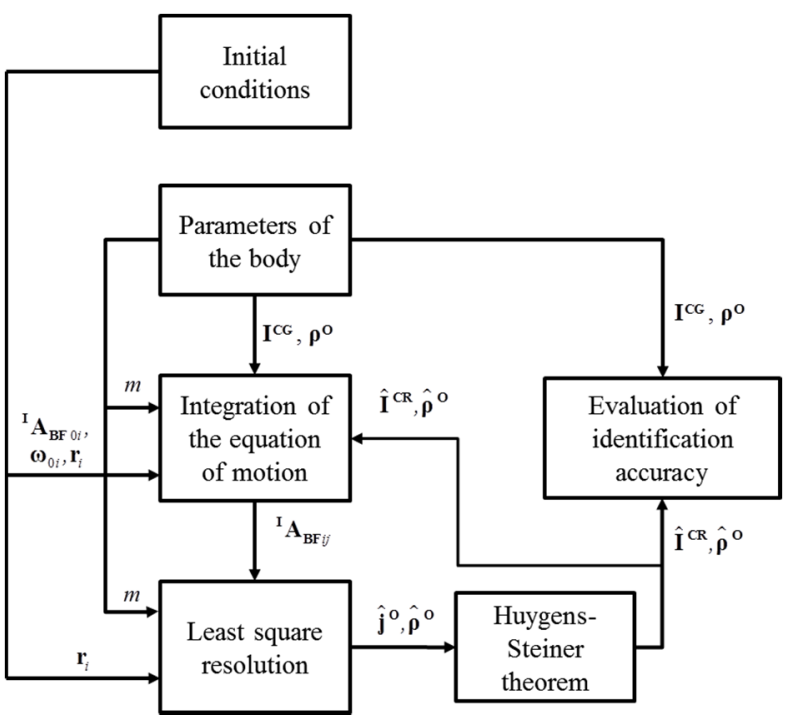

Figure 4. Simulation scheme of the identification of dynamic parameters. 
MATLAB are thus used to verify the algorithm efficiency. The MATLAB model consists of two parts: The simulation of the body dynamics and the identification of the dynamic parameters (Fig. 4).

The rotating body dynamics is described by (7). The simulation of the body motion requires to solve this equation to determine the angular position of the body, described by the rotation matrix ${ }^{\mathbf{I}} \mathbf{A}_{\mathbf{B F}}$. Finding ${ }^{\mathbf{I}} \mathbf{A}_{\mathbf{B F}}$ emulates data that will be obtained from the sensors of the test bench during the real experiments.

The part of the MATLAB model, dedicated to the identification of the inertial parameters, is similar to the one that will be used during the real experiments. The accuracy of the identification process can be estimated by comparing the "real" values $\mathbf{I}^{\mathbf{C R}}$ and $\boldsymbol{\rho}^{\mathbf{O}}$, chosen by the user for the current model run, and the values $\hat{\mathbf{I}}^{\mathbf{C R}}$ and $\hat{\boldsymbol{\rho}}^{\mathbf{o}}$ obtained by the identification process.

\section{E. Simulation of the body motion}

Equation (7) shall be written in the following form to be solved by the MATLAB Ordinary Differential Equation (ODE) solver

$$
\dot{\mathbf{y}}=f(\mathbf{y}),
$$

where $\mathbf{y}$ is the vector of unknowns. Equation (7) contains first and second order derivatives of the angular position of the body. Moreover, the angular velocity is not linearly dependent on the sought-for rotation matrix ${ }^{\mathbf{I}} \mathbf{A}_{\mathbf{B F}}$. The following solution is proposed to deal with this issue.

Let $\mathbf{y}$ to be the vector composed of the components of ${ }^{\mathrm{I}} \mathbf{A}_{\mathrm{BF}}$ and ${ }^{\mathrm{I}} \boldsymbol{\omega}$

$$
\mathbf{y}=\left[\begin{array}{lllllllll:lll}
A_{11} & A_{21} & A_{31} & A_{12} & A_{22} & A_{32} & A_{31} & A_{32} & A_{33} & \omega_{1} & \omega_{2} & \omega_{3}
\end{array}\right]^{T}
$$

Using (3), the first 9 components of $\dot{\mathbf{y}}$ can be found from the equation

$$
{ }^{\mathrm{I}} \dot{\mathbf{A}}_{\mathrm{BF}}=\left[{ }^{\mathrm{I}} \boldsymbol{\omega}\right]_{\times}{ }^{\mathrm{I}} \mathbf{A}_{\mathrm{BF}}
$$

Equation (7) can be solved for ${ }^{\mathrm{I}} \dot{\boldsymbol{\omega}}$, which are the last 3 components of $\dot{\mathbf{y}}$

$$
{ }^{\mathrm{I}} \dot{\boldsymbol{\omega}}={ }^{\mathrm{I}} \mathbf{A}_{\mathrm{BF}}\left(\left(\mathbf{I}^{\mathrm{CR}}\right)^{-1}\left({ }^{\mathrm{BF}} \mathbf{A}_{\mathbf{I}}{ }^{\mathrm{I}} \boldsymbol{\omega} \times \mathbf{I}^{\mathrm{CR}}{ }_{\mathrm{BF}} \mathbf{A}_{\mathbf{I}}{ }^{\mathrm{I}} \boldsymbol{\omega}-m\left[{ }^{\mathrm{BF}} \mathbf{A}_{\mathbf{I}}{ }^{\mathrm{I}} \mathbf{g}\right]_{\times} \boldsymbol{\rho}\right)\right)
$$

Thereby, the equations of motion can be expressed in the form (27) which is required by the ODE solver.

\section{F. Results}

MATLAB simulations were run for CAD models of $1 \mathrm{U}$ and $3 \mathrm{U}$ CubeSats with different dynamic characteristics and initial conditions, represented in Table I and Table III. At the beginning of each experiment, the CubeSat is at rest and the axes of $\mathcal{R}_{b f}$ and $\mathcal{R}_{I}$ are perfectly aligned.

The scaling coefficients for $\mathbf{r}$ and the duration of each experiment, which depends on the magnitude of $\boldsymbol{\rho}^{\mathbf{o}}$ derived during the previous iteration, were found empirically and are given in Table II. The sampling time for all iterations is 0.1 sec.
TABLE I. INITIAL CONDITIONS

\begin{tabular}{|l|c|c|c|c|}
\hline $\begin{array}{l}\text { Experiment } \\
\text { number, } \boldsymbol{p}\end{array}$ & $\mathbf{1}$ & $\mathbf{2}$ & $\mathbf{3}$ & $\mathbf{4}$ \\
\hline Initial offset, & {$\left[\begin{array}{l}0.02 \\
0.02 \\
0.02\end{array}\right]$} & {$\left[\begin{array}{c}-0.02 \\
-0.02 \\
0.02\end{array}\right]$} & {$\left[\begin{array}{c}0.02 \\
0.02 \\
-0.02\end{array}\right]$} & {$\left[\begin{array}{c}-0.02 \\
-0.02 \\
-0.02\end{array}\right]$} \\
\hline $\mathbf{r}$ (m) &
\end{tabular}

The results of the identification and the corresponding number of iterations, required to obtain $\boldsymbol{\rho}^{\mathbf{O}}$ with an accuracy of $1 \mu \mathrm{m}$ (that is needed to meet disturbing torque requirements) are given in Table 3.

These simulations provide good results in the identification of the $\mathrm{CM}$ location (largest error $<0.1 \%$ ) and of the moments of inertia (largest error $<1 \%$ ), but for the accurate identification of the products of inertia some additional measures might be needed. The latter can be explained by the small magnitudes of the products of inertia with respect to other identified values. However, the products of inertia should always be small due to the parallelepiped shape of the satellite. Furthermore, they are not important to the CubeSat balancing.

Based on the conducted simulations of the identification process, better results are obtained when at least 4 experiments are made such that, in each experiment, the geometric center of the CubeSat is located in a different octant of $\mathcal{R}_{I}$. However, further research on the optimization of the identification process shall be done to improve its efficiency.

TABLE II. SCALING COEFFICIENTS

\begin{tabular}{|c|c|c|}
\hline $\begin{array}{c}\boldsymbol{\rho}^{\mathbf{O}} \text { at the previous } \\
\text { iteration (m) }\end{array}$ & $\begin{array}{c}\text { Scaling coefficient } \\
\text { for } \mathbf{r}\end{array}$ & $\begin{array}{c}\text { Duration of each } \\
\text { experiment, } \boldsymbol{N}_{\boldsymbol{i}}(\mathbf{s e c})\end{array}$ \\
\hline$\geq 0.01$ & 1 & 5 \\
\hline$\geq 0.001$ & 0.5 & 10 \\
\hline$\geq 0.0001$ & 0.1 & 20 \\
\hline$<0.0001$ & 0.05 & 25 \\
\hline
\end{tabular}

TABLE III. RESULTS OF SimULATIONS

\begin{tabular}{|l|l|l|l|l|}
\hline \multirow{2}{*}{} & \multicolumn{2}{|c|}{ 1U CubeSat } & \multicolumn{2}{c|}{ 3U CubeSat } \\
\cline { 2 - 5 } & $\begin{array}{c}\text { CAD } \\
\text { values }\end{array}$ & $\begin{array}{c}\text { Identified } \\
\text { values }\end{array}$ & $\begin{array}{c}\text { CAD } \\
\text { values }\end{array}$ & $\begin{array}{c}\text { Identified } \\
\text { values }\end{array}$ \\
\hline$\rho_{1}^{\mathrm{O}}\left(\mathrm{m} \cdot 10^{-3}\right)$ & -0.2560 & -0.2558 & -1.6393 & -1.6398 \\
\hline$\rho_{2}^{\mathrm{O}}\left(\mathrm{m} \cdot 10^{-3}\right)$ & -0.9320 & -0.9317 & -1.2807 & -1.2814 \\
\hline$\rho_{3}^{\mathrm{o}}\left(\mathrm{m} \cdot 10^{-3}\right)$ & -9.9570 & -9.9572 & 17.1741 & 17.1749 \\
\hline $\mathrm{I}_{x \mathrm{x}}^{\mathrm{CM}}\left(\mathrm{kgm}^{2} \cdot 10^{-3}\right)$ & 1.5460 & 1.5325 & 30.6915 & 30.4716 \\
\hline $\mathrm{I}_{\mathrm{yy}}^{\mathrm{CM}}\left(\mathrm{kgm}^{2} \cdot 10^{-3}\right)$ & 1.5910 & 1.5797 & 29.6998 & 29.4381 \\
\hline $\mathrm{I}_{\mathrm{z}}^{\mathrm{CM}}\left(\mathrm{kgm}^{2} \cdot 10^{-3}\right)$ & 1.3840 & 1.3817 & 4.5775 & 4.5399 \\
\hline $\mathrm{I}_{\mathrm{xy}}^{\mathrm{CM}}\left(\mathrm{kgm}^{2} \cdot 10^{-3}\right)$ & 0.0090 & -0.0087 & 0.0250 & -0.2039 \\
\hline $\mathrm{I}_{\mathrm{xz}}^{\mathrm{CM}}\left(\mathrm{kgm}^{2} \cdot 10^{-3}\right)$ & -0.0070 & 0.0030 & -0.1459 & -0.0052 \\
\hline $\mathrm{I}_{\mathrm{yz}}^{\mathrm{CM}}\left(\mathrm{kgm}^{2} \cdot 10^{-3}\right)$ & 0.0060 & 0.0082 & 0.0030 & 0.0021 \\
\hline $\begin{array}{l}\mathrm{Number} \mathrm{of} \\
\text { iterations } \\
(\text { Section IV-D) }\end{array}$ & 4 & 4 & \\
\hline
\end{tabular}




\section{CONCLUSION}

A novel concept of an air bearing test bench for CubeSat ground testing was proposed in this paper. In contrast to existing air-bearing test benches, it allows unlimited 3 DoF rotations of the tested CubeSat while reducing the undesired influences caused by the other mobile elements attached to the CubeSat. Moreover, a dynamic parameter identification method, adapted to the proposed test bench, was presented. This method is mainly based on the sampling of free oscillating motions. Simulation results of dynamic parameter identification for CAD models were presented. Future work will aim at validating the identification method on the assembled test bench with a CubeSat prototype.

\section{REFERENCES}

[1] M. Swartwout, "The first one hundred CubeSats: a statistical look," Journal of Small Satellites, vol. 2, no. 2, pp. 213-233, 2013.

[2] J. Kim and B. Agrawal, "Automatic mass balancing of air-bearingbased three-axis rotational spacecraft simulator," Journal of Guidance, Control, and Dynamics, vol. 32, no. 3, pp. 1005-1017, May-June 2009.

[3] M. A. Peck and A. R. Cavender, "An airbearing-based testbed for momentum-control systems and spacecraft line of sight," Advances in the Astronautical Sciences (American Astronautical Society), pp. 427446, 2003.

[4] B. N. Agrawal and R. E. Rasmussen, "Air bearing based satellite attitude dynamics simulator for control software research and development," Proc.SPIE, Technologies for Synthetic Environments: Hardware-in-the-Loop Testing, vol. 4366, pp. 204-214, 2001.

[5] C. W. Crowell, Development and analysis of a small satellite attitude determination and control system testbed, Massachusetts, 2011.

[6] R. Fullmer, G. Peterson, W. Holmans, J. Smith, J. Nottingham, S. Anderson, T. Olsen and F. Redd, The development of a small satellite attitude control simulator, Utah State University, Loagan UT.

[7] G. Bisiacchi, J. Prado, L. Reyes, E. Vicente, F. Contreras, M. Mesinas and A. Juarez, "Three-axis air-bearing based platform for small satellite attitude determination and control simulation," Journal of Applied Research and Technology, vol. 3, no. 3, pp. 222-237, 2005.

[8] B. Kim, E. Velenis, P. Kriengsiri and P. Tsiotras, "Designing a lowcost spacecraft simulator," IEEE Control Systems Magazine, pp. 2637, August 2003.
[9] D. M. Meissner, "A three degrees of freedom test bed for nanosatellite and CubeSat attitude dynamics, determination, and control, " Monterey, California, 2009.

[10] D. Gallardo and R. Bevilacqua, "Six degrees of freedom experimental platform for testing autonomous satellites operations," in 8 th International ESA Conference on Guidance, Navigation and Control Systems, Karlovy Vary, 2011.

[11] T. Ustrzycki, R. Lee and H. Chesser, "Spherical air bearing attitude control simulator for nanosatellites," in AIAA Modeling and Simulation Technologies Conference, Portland, Oregon, 2011.

[12] J. L. Schwartz, The distributed spacecraft attitude control system simulator: from design concept to decentralized control, Blacksburg, Virginia, 2004.

[13] W. Khalil and E. Dombre, Modeling, Identification and Control of Robots, CRC Press, 2002.

[14] B. Siciliano, L. Sciavicco, L. Villani and G. Oriolo, Robotics. Modelling, Planning and Control, Springer, 2010.

[15] M. Gautier and S. Briot, "New method for global identification of the joint driven gains of robots using a known payload mass," in IEEE/RSJ International Conference on Intelegent Robots and Systems, San-Francisco, CA, 2011.

[16] C. H. An, C. G. Atkeson and J. M. Hollerbach, "Estimation of inertial parameters of rigid body links of manipulators," Massachusetts Institute of Technology, Artificial Intelligence Laboratory, Massachusetts, 1986.

[17] E. Wilson, C. Lages and R. Mah, "On-line, gyro-based, mass-property identification for thruster-controlled spacecraft using recursive least squares," Proceedings of the 45th IEEE International Midwest Symposium on Curcuits and Systems, August 2002.

[18] M. Gautier and S. Briot, "Dynamic parameter identification of a 6 DOF industrial robot using power model," in IEEE International Conference on Robotics and Automation (ICRA), Karlsruhe, 2013.

[19] J. L. Schwartz, M. A. Peck and C. D. Hall, "Historical review of airbearing spacecraft simulators," Journal of Guidance, Control, and Dynamics, vol. 26, no. 14, pp. 513-522, 2003.

[20] P. Wang, J. Yee et F. Hadaegh, "Synchronised rotation of multiple autonomous spacesrafts with rule-based control: experimental study," Journal of Guidance, Control, and Dynamics, vol. 24, n 12, pp. 352359, 2001.

[21] The CubeSat Program, CubeSat Design Specification Rev.13, Cal Poly SLO, 2014. 